\title{
Tamoxifen: using pharmacogenetics to rediscover an old drug
}

\author{
A. H. Kamal ${ }^{a}$, M. P. Goetz ${ }^{\text {b }}$ \\ ${ }^{a}$ Department of Medicine, Mayo Clinic College of Medicine, Rochester, MN, USA; ${ }^{b}$ Department of \\ Oncology, Mayo Clinic College of Medicine, Rochester, MN, USA.
}

\begin{abstract}
Tamoxifen can be considered a classic 'pro-drug', requiring metabolic activation to elicit pharmacological activity. Our findings suggest that both genetic and drug-induced factors that alter CYP2D6 enzyme activity influence the clinical response to tamoxifen. Given the small differences in disease-free survival comparing tamoxifen to third-generation aromatase inhibitors, knowledge of the genetic and environmental factors that influence CYP2D6 enzyme activity may provide a robust tool to individualize the hormonal therapy to breast cancer.
\end{abstract}

Keywords: Aromatase inhibitors; Breast cancer; Endocrine therapy; Estrogen receptor; Tamoxifen

\section{Introduction}

Breast cancer is the most common female malignancy in the Western world. Two-thirds of all breast cancers are estrogen receptor (ER) positive, a phenotypic characteristic that is prognostic of diseasefree survival (DFS) and predictive of response to endocrine therapy.

While tamoxifen is still the primary endocrine therapy for the prevention and treatment of premenopausal breast cancer, aromatase inhibitors (Al) are now commonly administered for post-menopausal breast cancer. However, the small absolute gain in DFS (2-3\%) and the lack of an overall survival advantage observed in the head-to-head trials continue to fuel the debate as to the role of tamoxifen for post-menopausal breast cancer [1,2]. In addition, the repeated demonstration that sequencing of hormonal therapy prolongs survival

Correspondence to: Matthew P. Goetz, MD, Department of Oncology, Mayo Clinic, 200 First Street Southwest, Rochester, MN 55905, USA. E-mail: goetz.matthew@mayo.edu; Tel: +1 507284 4849; Fax: +1 507 2841803

Received: 08/07/07 Accepted: 03/09/07 $\mathrm{BCO} / 585 / 2007 / \mathrm{FO}$ compared to 5 years of tamoxifen $[3,4]$ has provided additional impetus for the continued use of tamoxifen in this setting.

While a plethora of tumor or somatic factors, including gene expression profiles [5-8], have been associated with tamoxifen resistance, the beststudied biomarkers (e.g. progesterone receptor, HER1, and HER2) appear to predict resistance to endocrine therapy in general (e.g. both tamoxifen and Al), rather than resistance to a specific drug $[9,10]$. However, recent discoveries regarding the pharmacology and pharmacogenetics of tamoxifen indicate that genetically inherited variation in the enzyme responsible for the metabolic activation of tamoxifen may account for a portion of 'de novo' or early tamoxifen resistance. In this review, we will focus on the tamoxifen metabolic pathway, the clinical implications for treatment of endocrine-positive breast cancer, and the use of pharmacogenetics as a tool to individualize endocrine therapy.

\section{Tamoxifen metabolism}

For over 30 years, multiple pre-clinical and clinical studies have demonstrated that tamoxifen and its metabolites competitively bind to the ER in both 
breast and non-breast tissues to produce a complex phenotype of both agonist and antagonist effects. While researchers have consistently demonstrated that tamoxifen has minimal anti-proliferative activity in vitro [11-14] and in breast tumor xenograft models $[11,15]$, paradoxically, clinically significant tumor responses are observed when tamoxifen is administered to women with estrogen-positive breast cancer. For example, the clinical response rate observed in tamoxifen neoadjuvant trials is $35-40 \%$ $[16,17]$. In spite of the wide interindividual variability in the concentrations of tamoxifen and its metabolites, previous studies have not demonstrated a link between the plasma concentrations of tamoxifen or its metabolites with clinical response $[18,19]$.

For many years, tamoxifen clinical activity was felt to be substantially related to $4-\mathrm{OH}$ tamoxifen $[20,21]$, a primary tamoxifen metabolite with a 100 fold greater affinity for ERs and 30-100-fold more potency than tamoxifen in suppressing estrogendependent cell proliferation [22,23]. However, human steady-state concentrations of $4-\mathrm{OH}$ tamoxifen are negligible (5-10 nanomolar $(\mathrm{nM}))$ compared to tamoxifen $(370 \mathrm{nM})$ or its most abundant metabolite, $\mathrm{N}$-desmethyl-tamoxifen [(NDM; $650 \mathrm{nM})]$ [24].

Recently, the characterization of a secondary metabolite, 4-OH N-desmethyl-tamoxifen (endoxifen), originally described nearly 20 years ago as 'metabolite Bx' by Lien and colleagues [25,26], has led to the premise that endoxifen plays a central role in the activity of tamoxifen. Compared to $4-\mathrm{OH}$ tamoxifen, endoxifen has equivalent potency in vitro in ER- $\alpha$ and ER- $\beta$ binding [13], in suppressing ER-dependent breast cancer proliferation $[13,27]$ and in global ER-responsive gene expression [28], but importantly is present at concentrations up to 10-fold higher than 4-OH tamoxifen [29]. Further studies evaluating the effect of tamoxifen and its metabolites on the anti-proliferative TGF $\beta$ signal transduction pathway in human breast cancer cells have demonstrated that only $4-\mathrm{OH}$ tamoxifen and endoxifen have significant anti-proliferative activity and were able to induce TGF $\beta 2$ and T $\beta$ RII in MCF-7 cells [14]. Thus, in summary, 4-OH tamoxifen and endoxifen have the greatest affinity towards the ER and are tamoxifen metabolites exhibiting the most significant anti-proliferative activity.

\section{Cytochrome P450 metabolism of tamoxifen}

Tamoxifen is extensively hepatically metabolized by the cytochrome P450 system to several primary and secondary metabolites [30-32]. The major metabolites of primary tamoxifen metabolism are NDM, $4-\mathrm{OH}$ tamoxifen, and $\alpha$-hydroxy-tamoxifen. NDM,

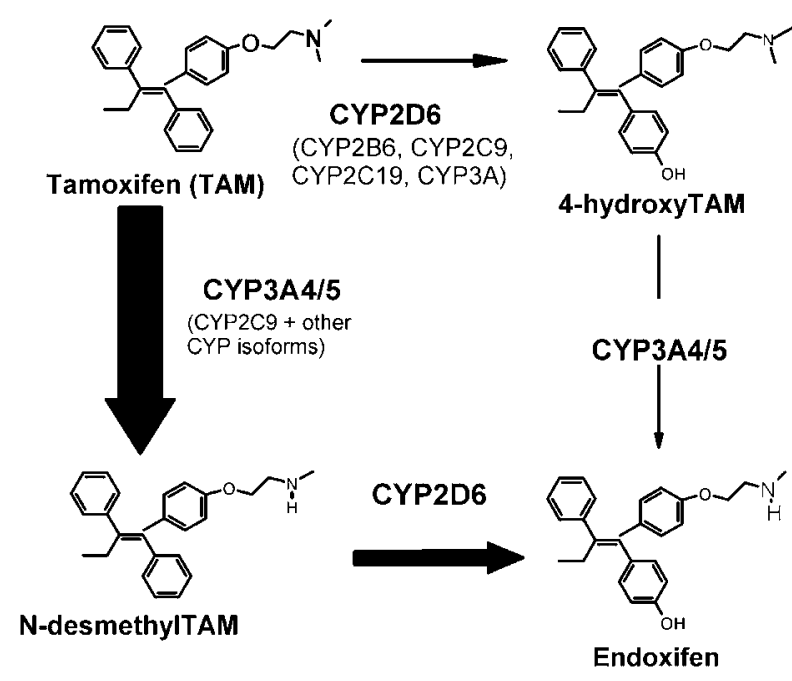

Figure 1.

Selected transformation pathways of tamoxifen and the main CYP enzymes involved. The relative contribution of each pathway to the overall oxidation of tamoxifen is shown by the thickness of the arrow, and the principal $P 450$ isoforms responsible are highlighted in larger fonts (modified in part from Jin et al. [24]).

resulting from the CYP3A4/5-mediated catalysis of tamoxifen, is quantitatively the major primary metabolite of tamoxifen, accounting for more than $90 \%$ of primary tamoxifen oxidation [32], whereas the steady-state concentrations of $4-\mathrm{OH}$ tamoxifen (accounting for less than 10\% of tamoxifen oxidation) are extremely low (5-10 nM) (Fig. 1)

Recent work by Desta et al. [32] has led to a more complete characterization of the secondary pathway of tamoxifen metabolism. NDM is predominantly biotransformed to $\alpha$-hydroxy $N$-desmethyl-, $\mathrm{N}$-didesmethyl-, and endoxifen. CYP2D6 is the rate-limiting enzyme responsible for the formation of endoxifen [32] (Fig. 1). In contrast to 4-OH tamoxifen, wherein multiple CYP450 isoforms are responsible for the oxidation of tamoxifen to 4-OH tamoxifen, CYP2D6 is the primary enzyme responsible for the catalysis of NDM to endoxifen $[32,33]$. Recent clinical studies have demonstrated that common CYP2D6 genetic variation (leading to reduced or absent CYP2D6 activity) or the inhibition of CYP2D6 activity significantly lowers the concentrations of endoxifen [24,33]. Specifically, plasma levels of endoxifen are up to 10-fold higher than $4-\mathrm{OH}$ tamoxifen, and range from $20 \mathrm{nM}$ in patients with no CYP2D6 activity (CYP2D6 poor metabolizers) up to $180 \mathrm{nM}$ in patients with normal or increased CYP2D6 activity (CYP2D6 extensive or ultra-rapid metabolizers) [29]. This makes endoxifen the most abundant active tamoxifen metabolite. 


\section{CYP2D6 and genetic variation}

The CYP2D6 gene is localized on chromosome 22q13.1. The genetic polymorphism of CYP2D6 is documented by the fact that 63 different major polymorphic CYP2D6 alleles are known, many of which are associated with increased, decreased, or abolished function of the final gene product. Some of the most common and important variant alleles distributed in different ethnic groups are listed in Table 1, and all variant alleles are presented at the home page of the human CYP allele nomenclature committee (http://www.imm.ki.se/cypalleles/cyp2d6. $\mathrm{htm})$. Carriers of any two of about 20 known null alleles are phenotypic poor metabolizers, representing about $5-10 \%$ of the individuals of European and North American Caucasian populations. Individuals at the higher end of the activity spectrum (ultra-rapid metabolizers) carry alleles with gene duplications and multiduplications, which lead to higher CYP2D6 expression and enzyme activity.

\section{Tamoxifen pharmacogenomics and breast cancer relapse}

Knowing that CYP2D6 is the rate-limiting enzyme responsible for the production of endoxifen and, additionally, contributes to the production of $4-\mathrm{OH}$ tamoxifen, we hypothesized that genetic variation and/or drug-induced variation in CYP2D6 leading to the poor metabolizer state could alter breast cancer outcomes in women administered tamoxifen. We performed a retrospective analysis of a prospective adjuvant tamoxifen trial (NCCTG 89-30-52) in postmenopausal women with surgically resected ERpositive breast cancer (stages I-III). Because of the difficulty in amplifying DNA from formalin-fixed paraffin-embedded tissue, only the CYP2D6 * 4 (the most common null allele contributing to the poormetabolizer state) and the ${ }^{*} 6$ (a rare allele with a frequency less than $1 \%$ ) were studied. The * 4 and *6 alleles account for $75-80 \%$ of CYP2D6 poor metabolizers in a predominantly Caucasian population [35]. As expected, no *6 variants were detected. Women with the CYP2D $6{ }^{*} 4 /{ }^{*} 4$ genotype had shorter relapse-free time and worse DFS compared with either the * $4 /$ wt or the wt/wt genotype (log rank $P=0.030$ and $P=0.020$, respectively). In a multivariate analysis, ${ }^{*} 4 /{ }^{*} 4$ patients still tended to have worse DFS (HR 1.86, $P=0.089$ ) [36]. Additionally, we observed differences in the incidence of moderate or severe hot flashes in patients homozygous for the ${ }^{*} 4$ allele $(0 \%)$ vs. those with 1 or 2 functional alleles $(20 \%)(P=0.06)$. These data supported the hypothesis that the CYP2D6mediated formation of the potent anti-estrogens may lead not only to differences in response but also to side-effects.

\section{CYP2D6 inhibitors and their effect on tamoxifen activation}

The measurement of enzyme activity for phase I and II drug metabolizing enzymes is complex, as genetic variation accounts for only one aspect of the known wide variability in the expression and activity of these enzymes. In the case of CYP2D6, many drugs, including the newer antidepressant drugs such as the selective serotonin reuptake inhibitors (SSRIs) and the serotonin and norepinephrine reuptake inhibitors (SNRIs), are commonly administered with tamoxifen to treat depression or to alleviate hot flashes [37-41]. Many SSRIs used to treat symptoms of hot flashes in tamoxifen-treated women are known inhibitors of the CYP2D6 enzyme [42]. Stearns et al. demonstrated that co-administration of paroxetine in tamoxifen-treated women dramatically reduced the plasma concentrations of endoxifen by nearly $65 \%$ in patients with normal CYP2D6 metabolism [33]. A follow-up prospective trial demonstrated that the co-administration of potent inhibitors of CYP2D6 (paroxetine and fluoxetine) and weak CYP2D6 inhibitors significantly lowered plasma concentrations of endoxifen [29].

Table 1. Major CYP2D6 alleles, effect on enzyme metabolism, and allele frequencies in selected populations.

\begin{tabular}{lllll}
\hline & & \multicolumn{2}{l}{ Allele frequencies (\%) } \\
\cline { 3 - 5 } Major variant alleles & Consequence & Caucasians & Asians & Black Africans \\
\hline CYP2D6*2xn & Increased enzyme activity & $1-5$ & $0-2$ & 2 \\
CYP2D6*4 & Inactive enzyme & $12-21$ & 1 & 2 \\
CYP2D6*5 & No enzyme & $2-7$ & 6 & 4 \\
CYP2D6*10 & Decreased activity & $1-2$ & 51 & 6 \\
CYP2D6*17 & Decreased activity & 0 & 0 & $20-35$ \\
CYP2D6*41 & Decreased activity & $8-10$ & $0-2$ & $11-14$ \\
\hline
\end{tabular}

Modified from Ingelman-Sundberg [34]. 


\section{CYP2D6 metabolism and tamoxifen treatment outcome}

We recently updated our analysis of the NCCTG 8930-52 tamoxifen trial by performing a comprehensive analysis of the combined effect of exogenous inhibition of the CYP2D6 enzyme and genetic variation on breast cancer outcomes [43]. We reviewed the medical records at each NCCTG randomizing site to determine whether the following known CYP2D6 inhibitors were co-administered with tamoxifen: fluoxetine, paroxetine, sertraline, cimetidine, amiodarone, doxepin, ticlopidine, and haloperidol. In our analysis, we used the in vivo data regarding the known combined effect of CYP2D6 genotype and CYP2D6 inhibitors on endoxifen plasma concentrations [24,29]. 'Extensive' metabolizers were women without a CYP2D6 *4 allele, who did not receive a CYP2D6 inhibitor. 'Decreased' CYP2D6 metabolism was defined as patients with one or two * 4 alleles or the confirmation that a CYP2D6 inhibitor was co-administered with tamoxifen (regardless of genotype).

Medication history was available in 225/256 eligible patients and we confirmed that 13 patients (6\%) were co-prescribed a CYP2D6 inhibitor with a median duration of 2-3 years. Knowledge of both medication history and CYP2D6 genotype (available in 180 pts) allowed us to classify patients as having 'extensive' $(n=115)$ or 'decreased' $(n=65)$ CYP2D6 metabolism. In a multivariate analysis, patients with 'decreased' metabolism had worse relapse-free survival (RFS) $(P=0.017 ; \mathrm{HR}=1.74)$, relative to patients with 'extensive' metabolism. Additionally, based on the in vivo data regarding the combined effect of CYP2D6 genotype and CYP2D6 inhibitors on endoxifen plasma concentrations [24,29], we further classified patients with 'decreased' CYP2D6 metabolism into 'intermediate' or 'poor' metabolizer categories, based on the potency of the CYP2D6 inhibitor prescribed. While women classified as having 'intermediate' metabolism tended to exhibit worse RFS (HR 1.63, $P=0.075)$, patients classified as 'poor metabolizers' had the worst outcome, with significantly worse RFS (HR 2.69, $P=0.005$ ) [43], consistent with a gene dosage effect (Fig. 2).

Recently, our findings were validated by Schroth et al. [44], who demonstrated the significance of CYP2D6 genetic variation in a retrospective cohort of 206 tamoxifen-treated and 280 tamoxifen-untreated breast cancer patients. Tamoxifentreated women with one or two CYP2D6 alleles associated with absent or reduced enzyme function $\left({ }^{*} 4,{ }^{*} 5,{ }^{*} 10,{ }^{*} 41\right)$ had significantly more recurrences, shorter relapse-free time $(\mathrm{HR}=2.24 ; 95 \% \mathrm{Cl}$, 1.16-4.33; $P=0.02)$, and worse event-free survival

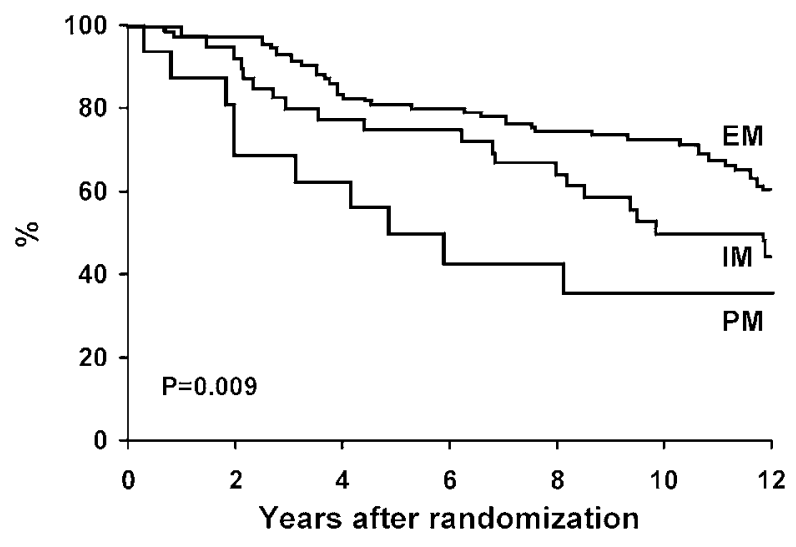

Figure 2.

Kaplan-Meier estimates of relapse-free survival based on metabolizer status (extensive, intermediate, or poor) (reprinted from Goetz et al. [43]).

$(\mathrm{HR}=1.89 ; 95 \% \mathrm{Cl}, 1.10-3.25 ; P=0.02)$ compared to carriers of functional alleles [44]. As expected, there was no association between treatment outcome and CYP2D6 genotype in the cohort of patients that did not receive tamoxifen.

\section{Tamoxifen and FDA relabeling}

On October 18, 2006, an FDA Advisory Subcommittee was convened to review the tamoxifen research findings to date and to make a recommendation regarding a label change. The consensus of the Subcommittee was that the label should be updated to reflect the fact that postmenopausal women with ER-positive breast cancer who are CYP2D6 poor metabolizers treated with tamoxifen (by genotype or drug interaction) are at increased risk for breast cancer recurrence.

\section{Clinical impact of tamoxifen pharmacogenomics}

One of the consistent findings in tamoxifen adjuvant studies to date is the demonstration of a peak in the annual hazard rates of recurrence within the first 2-3 years of initiating tamoxifen [45]. In our study, by grouping patients according to metabolizer status, the 2-year RFS rates by metabolizer status were $98 \%, 92 \%$, and $68 \%$ for extensive, intermediate, and poor metabolizers, respectively (Fig. 2). Furthermore, when comparing instantaneous hazard rates for RFS by CYP2D6 enzyme status (Fig. 3), our data demonstrate an immediate broad peak in the RFS hazard rate for patients with 'decreased' metabolism while the hazard rate in patients with extensive metabolism was reduced and did not peak until nearly the 4th year. These 


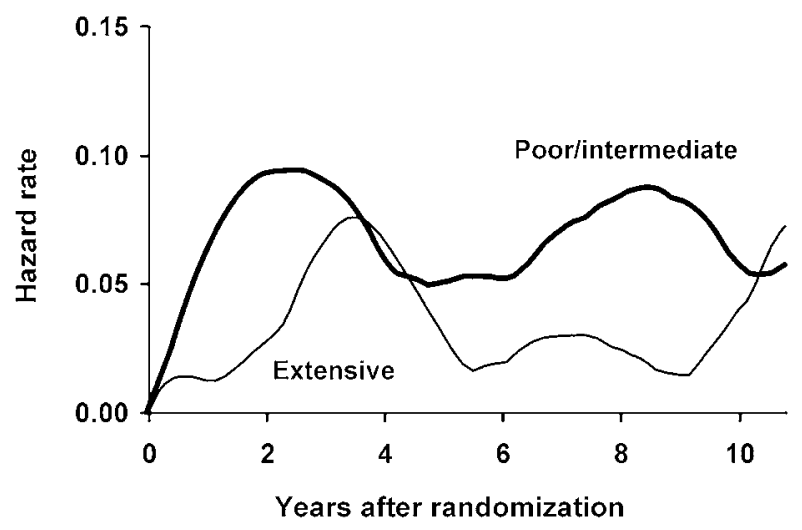

Figure 3.

Smoothed Hazard rates for relapse-free survival comparing patients with extensive vs. decreased CYP2D6 metabolism (reprinted from Goetz et al. [43]).

findings suggest that the peak in the annual hazard rates for recurrence in tamoxifen-treated women may be related to genetic or drug-induced variation in CYP2D6. Furthermore, our findings suggest that patients with decreased CYP2D6 metabolism who have not relapsed within the first 2-3 years of receiving tamoxifen therapy may be the subset that derives the greatest benefit from switching to an Al.

Our data may have profound implication for the long-term endocrine management of breast cancer, which is characterized by an unrelenting risk of relapse that carries well into the 2nd decade following diagnosis [46]. Notably, CYP2D6 is not known to be involved in the metabolism of any of the Al. In this way, CYP2D6 genotyping may identify patients most suitable for sequencing of hormonal therapy. Given the potential implications of longterm estrogen deprivation as is currently being evaluated in studies looking at 10 years of $\mathrm{Al}$, a strategy of sequencing may provide the most tolerable and efficacious strategy if CYP2D6 pharmacogenetics is validated as an early predictor or tamoxifen relapse in the head-to-head trials.

\section{Current management of ER-positive breast cancer}

It is our practice to fully inform patients of the importance of CYP2D6 as a key activator of tamoxifen. We consider CYP2D6 genotyping in settings wherein alternative therapies are known to be equivalent or superior to tamoxifen monotherapy (e.g. post-menopausal adjuvant breast cancer). However, it is important to stress that further research is needed to determine the optimal sequence of hormonal therapy (tamoxifen for some duration followed by an $\mathrm{Al}$ ) in patients who carry one or two functional CYP2D6 alleles. For pre-menopausal breast cancer, there are no published data regarding CYP2D6 genotype and treatment outcome. In the setting of chemoprevention, a small case-control study from the Italian chemoprevention group demonstrated a higher likelihood that tamoxifen-treated women homozygous for the *4 allele would develop breast cancer compared to those with the wild-type genotype [47]. These data are considered preliminary, and further research is needed in pre-menopausal women before using CYP2D6 genotype to exclude patients from tamoxifen.

Additionally, we recommend a careful evaluation of a patient's prescription and non-prescription medications, with a recommendation for discontinuation of potent CYP2D6 inhibitors. Regarding the non-hormonal treatment of hot flashes, we remind women that hot flashes may be a biomarker of tamoxifen drug effect [48]. In this setting, administration of an SSRI or SNRI like venlafaxine with little or no effect on endoxifen plasma levels [29] appears to be a reasonable treatment of tamoxifen-induced hot flashes.

In conclusion, our findings suggest that both genetic and drug-induced factors which alter CYP2D6 enzyme activity influence the clinical response to tamoxifen. Given the small differences in DFS comparing tamoxifen to third-generation Als, knowledge of the genetic and environmental factors that influence CYP2D6 enzyme activity may provide a robust tool to individualize the hormonal therapy of breast cancer.

\section{Acknowledgements}

Supported in part by CA 90628: Paul Calabresi Program in Clinical-Translational Research at Mayo Clinic (MPG); CA 116201: Career Development Award from Mayo Cancer Center Breast Cancer SPORE (MPG); and U10 GM31388-6: Pharmacogenetics of Phase II Drug Metabolizing Enzymes Pharmacogenomic Clinic Study Core (MPG).

\section{References}

1. Coates AS, Keshaviah A, Thurlimann B, et al. Five years of letrozole compared with tamoxifen as initial adjuvant therapy for postmenopausal women with endocrineresponsive early breast cancer: update of study BIG 1-98. J Clin Oncol 2007; 25: 486-492.

2. Howell A, Cuzick J, Baum M, et al. Results of the ATAC (Arimidex, Tamoxifen, Alone or in Combination) trial after completion of 5 years' adjuvant treatment for breast cancer. Lancet 2005; 365: 60-62.

3. Jonat W, Gnant M, Boccardo F, et al. Switching from adjuvant tamoxifen to anastrozole in postmenopausal women with hormone-responsive early breast cancer: a 
meta-analysis of the ARNO 95 trial, ABCSG trial 8, and the ITA trial. Breast Can Res Treat 2005.

4. Coombes RC, Paridaens R, Jassem J, et al. First mature analysis of the Intergroup Exemestane Study (IES). J Clin Oncol 2006; 24(18S): LBA527.

5. Ma XJ, Wang Z, Ryan PD, et al. A two-gene expression ratio predicts clinical outcome in breast cancer patients treated with tamoxifen. Cancer Cell 2004; 5: 607-616.

6. Paik S, Shak S, Tang G, et al. A multigene assay to predict recurrence of tamoxifen-treated, nodenegative breast cancer. N Engl J Med 2004; 351(27): 2817-2826.

7. Goetz MP, Suman VJ, Ingle JN, et al. A two-gene expression ratio of homeobox 13 and interleukin-17B receptor for prediction of recurrence and survival in women receiving adjuvant tamoxifen. Clin Cancer Res 2006; 12(7): 2080-2087.

8. Jansen MP, Foekens JA, van Staveren IL, et al. Molecular classification of tamoxifen-resistant breast carcinomas by gene expression profiling. J Clin Oncol 2005; 23: 732-740.

9. Viale G, Regan M, Dell'Orto P, et al. Central review of ER, $\mathrm{PgR}$, and HER-2 in BIG 1-98 evaluating letrozole vs. tamoxifen as adjuvant endocrine therapy for postmenopausal women with receptor-positive breast cancer. San Antonio Breast Cancer Symposium, 2006.

10. Dowsett $M$, Allred $C$, et al. Relationship between quantitative ER and PgR expression and HER2 status with recurrence in the ATAC trial. Breast Can Res Treat 2006; 100: 12 [Abstract 48].

11. Brunner N, Bronzert D, Vindelov LL, et al. Effect on growth and cell cycle kinetics of estradiol and tamoxifen on MCF-7 human breast cancer cells grown in vitro and in nude mice. Cancer Res 1989; 49: 1515-1520.

12. Johnson MD, Westley BR, May FE. Oestrogenic activity of tamoxifen and its metabolites on gene regulation and cell proliferation in MCF-7 breast cancer cells. $\mathrm{Br} \mathrm{J}$ Cancer 1989; 59: 727-738.

13. Johnson MD, Zuo $\mathrm{H}$, Lee $\mathrm{KH}$, et al. Pharmacological characterization of 4-hydroxy- $N$-desmethyl tamoxifen, a novel active metabolite of tamoxifen. Breast Cancer Res Treat 2004; 85: 151-159.

14. Buck MR, Coller JK, Murdter TE, et al. TGFb2 and TbRII are valid molecular biomarkers for the antiproliferative effects of tamoxifen and tamoxifen metabolites in breast cancer cells. Breast Can Res Treat, 2007, on-line first, DOI: 10.1007/s10549-007-9526-7.

15. Long BJ, Jelovac D, Handratta V, et al. Therapeutic strategies using the aromatase inhibitor letrozole and tamoxifen in a breast cancer model. J Natl Cancer Inst 2004; 96: 456-465.

16. Ellis MJ, Coop A, Singh B, et al. Letrozole is more effective neoadjuvant endocrine therapy than tamoxifen for ErbB-1- and/or ErbB-2-positive, estrogen receptorpositive primary breast cancer: evidence from a phase III randomized trial. J Clin Oncol 2001; 19: 3808-3816.

17. Smith IE, Dowsett M, Ebbs SR, et al. Neoadjuvant treatment of postmenopausal breast cancer with anastrozole, tamoxifen, or both in combination: the Immediate Preoperative Anastrozole, Tamoxifen, or
Combined with Tamoxifen (IMPACT) multicenter double-blind randomized trial. J Clin Oncol 2005; 23: 5108-5116.

18. Bratherton DG, Brown $\mathrm{CH}$, Buchanan $\mathrm{R}$, et al. $\mathrm{A}$ comparison of two doses of tamoxifen (Nolvadex) in postmenopausal women with advanced breast cancer: $10 \mathrm{mg}$ bd versus $20 \mathrm{mg}$ bd. $\mathrm{Br} J$ Cancer 1984; 50: 199-205.

19. MacCallum J, Cummings J, Dixon JM, et al. Concentrations of tamoxifen and its major metabolites in hormone responsive and resistant breast tumours. $\mathrm{Br} J$ Cancer 2000; 82: 1629-1635.

20. Jordan VC, Collins MM, Rowsby L, et al. A monohydroxylated metabolite of tamoxifen with potent antioestrogenic activity. J Endocrinol 1977; 75: 305-316.

21. Jordan VC. Metabolites of tamoxifen in animals and man: identification, pharmacology, and significance. Breast Cancer Res Treat 1982; 2: 123-138.

22. Borgna JL, Rochefort H. Hydroxylated metabolites of tamoxifen are formed in vivo and bound to estrogen receptor in target tissues. J Biol Chem 1981; 256: 859-868.

23. Robertson DW, Katzenellenbogen JA, Long DJ, et al. Tamoxifen antiestrogens: a comparison of the activity, pharmacokinetics, and metabolic activation of the cis and trans isomers of tamoxifen. J Steroid Biochem. 1982; 16: 1-13.

24. Jin Y, Desta Z, Stearns V, et al. CYP2D6 genotype, antidepressant use, and tamoxifen metabolism during adjuvant breast cancer treatment. $J$ Natl Cancer Inst 2005; 97: 30-39.

25. Lien EA, Solheim E, Lea OA, et al. Distribution of 4-hydroxy- $N$-desmethyltamoxifen and other tamoxifen metabolites in human biological fluids during tamoxifen treatment. Cancer Res 1989; 49: 2175-2183.

26. Lien EA, Anker G, Lonning PE, et al. Decreased serum concentrations of tamoxifen and its metabolites induced by aminoglutethimide. Cancer Res 1990; 50: 5851-5857.

27. Lim YC, Desta Z, Flockhart DA, et al. Endoxifen (4-hydroxy- $N$-desmethyl-tamoxifen) has anti-estrogenic effects in breast cancer cells with potency similar to 4-hydroxy-tamoxifen. Cancer Chemother Pharmacol 2005; 55: 471-478.

28. Lim YC, Li L, Desta Z, et al. Endoxifen, a secondary metabolite of tamoxifen, and 4-OH-tamoxifen induce similar changes in global gene expression patterns in MCF-7 breast cancer cells. J Pharmacol Exp Ther 2006; 318: $503-512$.

29. Borges S, Desta Z, Li L, et al. Quantitative effect of CYP2D6 genotype and inhibitors on tamoxifen metabolism: implication for optimization of breast cancer treatment. Clin Pharmacol Ther 2006; 80: 61-74.

30. Lonning PE, Lien EA, Lundgren S, et al. Clinical pharmacokinetics of endocrine agents used in advanced breast cancer. Clin Pharmacokinet 1992; 22: 327-358.

31. Crewe HK, Ellis SW, Lennard MS, et al. Variable contribution of cytochromes P450 2D6, 2C9 and 3A4 to the 4-hydroxylation of tamoxifen by human liver microsomes. Biochem Pharmacol 1997; 53: 171-178. 
32. Desta Z, Ward BA, Soukhova NV, et al. Comprehensive evaluation of tamoxifen sequential biotransformation by the human cytochrome P450 system in vitro: prominent roles for CYP3A and CYP2D6. J Pharmacol Exp Ther 2004; 310(3): 1062-1075.

33. Stearns $\mathrm{V}$, Beebe $\mathrm{KL}$, lyengar $\mathrm{M}$, et al. Paroxetine controlled release in the treatment of menopausal hot flashes: a randomized controlled trial. JAMA 2003; 289: 2827-2834.

34. Ingelman-Sundberg M. Genetic polymorphisms of cytochrome P450 2D6 (CYP2D6): clinical consequences, evolutionary aspects and functional diversity. Pharmacogenomics J 2005; 5: 6-13.

35. Sachse C, Brockmoller J, Bauer S, et al. Cytochrome P450 2D6 variants in a Caucasian population: allele frequencies and phenotypic consequences. Am J Hum Genet 1997; 60: 284-295.

36. Goetz MP, Rae JM, Suman VJ, et al. Pharmacogenetics of tamoxifen biotransformation is associated with clinical outcomes of efficacy and hot flashes. J Clin Oncol 2005; 23: 9312-9318.

37. Loprinzi CL, Kugler JW, Sloan JA, et al. Venlafaxine in management of hot flashes in survivors of breast cancer: a randomised controlled trial. Lancet 2000; 356: 2059-2063.

38. Loprinzi CL, Sloan JA, Perez EA, et al. Phase III evaluation of fluoxetine for treatment of hot flashes. J Clin Oncol 2002; 20: 1578-1583.

39. Stearns V, Johnson MD, Rae JM, et al. Active tamoxifen metabolite plasma concentrations after coadministration of tamoxifen and the selective serotonin reuptake inhibitor paroxetine. J Nat/ Cancer Inst 2003; 95: 1758-1764.

40. Kimmick GG, Lovato J, McQuellon R, et al. Randomized, double-blind, placebo-controlled, crossover study of sertraline (Zoloft) for the treatment of hot flashes in women with early stage breast cancer taking tamoxifen. Breast J 2006; 12: 114-122.

41. Barton DL, Loprinzi CL, Novotny P, et al. Pilot evaluation of citalopram for the relief of hot flashes. J Support Oncol 2003; 1: 47-51.

42. Hemeryck A, Belpaire FM. Selective serotonin reuptake inhibitors and cytochrome P-450 mediated drug-drug interactions: an update. Curr Drug Metab 2002; 3: 13-37.

43. Goetz MP, Knox SK, Suman VJ, et al. The impact of cytochrome P450 2D6 metabolism in women receiving adjuvant tamoxifen. Breast Cancer Res Treat 2007; 101: 113-121.

44 Schroth W, Antoniadou L, Fritz P, et al. Breast cancer treatment outcome with adjuvant tamoxifen in relation to Patient CYP2D6 and CYP2C19 genotypes. J Clin Oncol 2007, in Press.

45. Buzdar AU, Cuzick J. Anastrozole as an adjuvant endocrine treatment for postmenopausal patients with breast cancer: emerging data. Clin Cancer Res 2006; 12: 1037s-1048s.

46. Early Breast Cancer Trialists' Collaborative Group (EBCTCG). Effects of chemotherapy and hormonal therapy for early breast cancer on recurrence and 15-year survival: an overview of the randomised trials. Lancet 2005; 365: 1687-1717.

47. Bonanni B, Macis D, Maisonneuve P, et al. Polymorphism in the CYP2D6 tamoxifen-metabolizing gene influences clinical effect but not hot flashes: data from the Italian Tamoxifen Trial. J Clin Oncol 2006; 24: 3708-3709, author reply 3709.

48. Mortimer JE, Flatt SW, Parker BA, et al. Tamoxifen, hot flashes and recurrence in breast cancer. Breast Cancer Res Treat 2007; May 31; [Epub ahead of print] DOI: 10.1007/s10549-007-9612-X. 